\title{
TOTALLY UMBILICAL SUBMANIFOLDS OF A COMPLEX SPACE FORM
}

\section{SHAHID ALI AND VIQAR AZAM KHAN}

Recently Yamaguchi and others [3] have classified extrinsic spheres of a Kaehler manifold. Using their result, in this paper we classify the totally umbilical submanifolds of a complex space form. Our main result is the following:

Theorem: Let $M$ be an $n$-dimensional $(n>2)$ complete, simply connected, totally umbilical submanifold of a $2 m$-dimensional complex space form $\bar{M}(c)$. Then $M$ is one of the following

(i) A complex space form $M(c)$

(ii) Totally real submanifold of constsnt curvature

(iii) Isometric to an ordinary sphere

(iv) Homothetic to a sasakian manifold.

\section{Preliminaries}

Let $\bar{M}(c)$ be a $2 m$-dimensional complex space form i.e. a Kaehler manifold of constant holomorphic sectional curvature $c$. The curvature tensor $\bar{R}$ of $\bar{M}(c)$ is given by

$$
\bar{R}(X, Y) Z=\frac{c}{4}[g(Y, Z) X-g(X, Z) Y+g(J Y, Z) J X-g(J X, Z) J Y+2 g(X, J Y) J Z]
$$

Let $M$ be an $n$-dimensional submanifold of $M$. Then the Riemannian connection $\bar{\nabla}$ of $\bar{M}$ gives rise to a connection $\nabla$ on $M$ and a connection $\nabla^{\perp}$ in the normal bundle $\nu$ of $M$. The Gausś and Weingarten formulae are

$$
\begin{aligned}
& \bar{\nabla}_{X} Y=\nabla_{X} Y+h(X, Y), \\
& \bar{\nabla}_{X} N=-A_{N} X+\nabla_{X}^{\perp} N .
\end{aligned}
$$

where $X, Y$ are vector fields on $M, N \varepsilon \nu$ and $h, A_{N}$ are second fundamental forms connected by

$$
g(h(X, Y), N)=g\left(A_{N} X, Y\right) .
$$

The submanifold $M$ is said to be totally umbilical if

$$
h(X, Y)=g(X, Y) H
$$

Received February 7, 1988. 
where $H=\frac{1}{n}$ (trace $h$ ), is called the mean curvature vector. If $h=0$, then $M$ is said to be totally geodasic and if $H=0$, then $M$ is said to be minimal. For totally umbilical submanifold, they are equivalent. The equations of Gauss and Codazzi for totally umbilical submanifold in $\bar{M}(c)$ are

$$
\begin{aligned}
R(X, Y, Z, W)= & {\left[\frac{c}{4}+g(H, H)\right][g(Y, Z) g(X, W)-g(X, Z) g(Y, W)] } \\
& \left.+\frac{c}{4}[g(J Y, Z) g(J X, W)-g(J X, Z) g(J Y, W)+2 g(X, J Y) g(J Z, W(1)]\}\right) \\
{[\bar{R}(X, Y) Z]^{\perp}=} & g(Y, Z) \nabla_{X}^{\perp} H-g(X, Z) \nabla_{Y}^{\perp} H
\end{aligned}
$$

where []$^{\perp}$ denotes the normal component of $\bar{R}(X, Y) Z$.

\section{Proof of the theorem.}

As $n>2$, for every vector field $X$ on $M$ we can choose a vector field $Y$ on $M$ orthogonal to both $X$ and $J X$. Thus (1.7) gives

$$
[R(X, Y) Y]^{\perp}=g(Y, Y) \nabla \frac{\perp}{X} H
$$

On the other hand from (1.1) we have

$$
R(X, Y) Y=\frac{c}{4} g(Y, Y) X
$$

From (2.1) and (2.2) we get

$$
\nabla_{X}^{\frac{1}{X}} H=0, \text { for every vector } X \text { on } M \text {. }
$$

If $H=0$, then $M$ could be complex submanifold (as complex submanifolds of Kaehler manifold are minimal), we get part (i) of the theorem by (1.6).

If $H \neq 0$, then certainly $M$ is not complex submanifold but it is an extrinsic sphere by (2.3). Then rest of our theorem follows from [3].

\section{Acknowledgement}

The authors express their deep sense of gratitude to Prof. S.I. Husain for his kind help.

\section{References}

[1] B.Y. Chen, Geometry of Submanifolds, M. Dekker, New York, 1973.

[2] K. Ogiue, "Differential geometry of Kaehler Submanifolds," Advances in Math. 13(1974), 73-114.

[3] S. Yamaguchi, H. Menoto and N. Kawabata, "Extrinsic spheres in a Kaehler manifold," Michi. Math. J. 31, No. 1, 1984, 15-19. 